\title{
Healthcare Barriers of Refugees Post-resettlement
}

\author{
Meghan D. Morris · Steve T. Popper • \\ Timothy C. Rodwell $\cdot$ Stephanie K. Brodine $\cdot$ \\ Kimberly C. Brouwer
}

Published online: 25 August 2009

(C) The Author(s) 2009. This article is published with open access at Springerlink.com

\begin{abstract}
The majority of refugees spend the greater part of their lives in refugee camps before repatriation or resettlement to a host country. Limited resources and stress during residence in refugee camps can lead to a variety of acute and chronic diseases which often persist upon resettlement. However, for most resettled refugees little is known about their health needs beyond a health assessment completed upon entry. We conducted a qualitative pilotstudy in San Diego County, the third largest area in California, USA for resettling refugees, to explore health care access issues of refugees after governmental assistance has ended. A total of 40 guided in-depth interviews were conducted with a targeted sample of informants (health care practitioners, employees of refugee serving organizations, and recent refugee arrivals) familiar with the health needs of refugees. Interviews revealed that the majority of refugees do not regularly access health services. Beyond individual issues, emerging themes indicated that language and communication affect all stages of health care accessfrom making an appointment to filling out a prescription. Acculturation presented increased stress, isolation, and new responsibilities. Additionally, cultural beliefs about health care directly affected refugees' expectation of care. These barriers contribute to delayed care and may directly influence refugee short- and long-term health. Our findings
\end{abstract}

M. D. Morris $(\bowtie)$ - S. T. Popper · T. C. Rodwell ·

K. C. Brouwer

Department of Medicine, Division of Global Public Health, University of California, San Diego, 9500 Gilman Drive,

MC 0507, La Jolla, CA 93093-0507, USA

e-mail: mdmorris@ucsd.edu

S. K. Brodine

Graduate School of Public Health, San Diego State University, 5500 Campanile Drive, San Diego, CA 92182-4162, USA suggest the need for additional research into contextual factors surrounding health care access barriers, and the best avenues to reduce such barriers and facilitate access to existing services.

Keywords Refugee health · Needs assessment . Barriers to health care $\cdot$ San Diego

\section{Introduction}

In 2006, the United Nations High Commissioner for Refugees (UNHCR) reported that there were approximately 33 million displaced people worldwide [1]. Among the 9 million refugees resettled that year, the United States (U.S.) hosted 844,000 [1]. Refugees-defined by the UNHCR as individuals who have been forcibly displaced outside their native countries-come from a history of hardship, including war, famine, and violence. They are often faced with a variety of acute and chronic diseases resulting from difficult conditions in refugee camps and incomplete medical care prior to resettlement [2]. High rates of tuberculosis, malaria, hepatitis, intestinal parasites, and nutritional deficiencies have been documented in resettled refugees [3-8]. Whether these conditions persist post-resettlement or new ones emerge is not fully understood as health of resettled refugees is seldom assessed in the years after arrival in a host country.

In the U.S., treatment for infectious diseases, such as tuberculosis and parasites, is provided by health care personnel from county public health departments during a 'domestic health assessment' shortly after arrival. However, in most resettlement sites scant attention is devoted to chronic and mental health conditions. Refugees resettled in the U.S. have 8 months to become economically 
independent before cash assistance from the U.S. government terminates and they then become subject to standard eligibility requirements of Medicaid (joint Federal-State health insurance program for low-income families). Few studies have examined utilization of health care services after governmental assistance has ended and how this might relate to post-resettlement health conditions. Increased understanding of health needs and potential barriers to accessing health care of these resettled refugees is a critical priority for better allocation of sparse health care funds and improving refugee health.

Between 2001 and 2005, 5,090 refugees arrived in San Diego, making it the third largest resettlement site in the U.S. state of California [9]. The San Diego refugee population is comprised of countless ethnicities and cultures and includes a diversity of educational backgrounds, language abilities, and ages [10]. This poses unique challenges to informing newly arrived refugees about health care options. A recent survey of members of the San Diego Refugee Forum, an affiliation of refugee service providers, found that $56 \%$ of its members ranked health or health care as the most important issue facing refugees during their initial years in the U.S. [11].

Refugees are generally resettled to nations with economic opportunities and health resources which are vast in comparison to that of refugee camps. Whether they are fully able to utilize the resources available to them upon resettlement is uncertain. Additionally, refugee health conditions emerging in the years post-resettlement are understudied. This assessment focused on eliciting qualitative information from informants on health conditions and health care access of refugees post-resettlement. Study aims were focused on understanding the structural and social barriers affecting resettled refugees from fully accessing health services. The barriers perceived as having the greatest impact on access to health care in our study population are presented.

\section{Methods}

\section{Study Population}

The majority of those resettled to San Diego County from 2000 to 2004 were younger than 25-years-old, and half were female (see Table 1). Most of the refugees arriving in San Diego from 2001 to 2005 originated from just eight countries: Somalia $(27 \%)$, Iraq $(15 \%)$, Vietnam $(8 \%)$, Sudan (7\%), Iran (7\%), Afghanistan (6\%), Ethiopia (6\%) and Russia (4\%) [10]. Guided in-depth interviews were conducted with 40 informants in San Diego County, CA from December 2006 through March 2007. Participants fell into one of four categories: (1) refugees who had resettled
Table 1 Demographics of recent refugee arrivals in San Diego (2001-2005)

Source: San Diego arrivals by groups and nationalities, 20012005 (2006). San Diego County: California department of health services refugee health section

\begin{tabular}{|c|c|}
\hline Category & $\%$ \\
\hline \multicolumn{2}{|l|}{ Age (years) } \\
\hline$<15$ & 29 \\
\hline $15-24$ & 27 \\
\hline $25-34$ & 17 \\
\hline $35-44$ & 12 \\
\hline$>45$ & 15 \\
\hline \multicolumn{2}{|l|}{ Gender } \\
\hline Male & 52 \\
\hline \multicolumn{2}{|l|}{ Status } \\
\hline Primary refugee & 76 \\
\hline Secondary migrant & 2 \\
\hline Asylee & 21 \\
\hline Parolee & 1 \\
\hline Victim of trafficking & $<1$ \\
\hline \multicolumn{2}{|l|}{ Country of origin } \\
\hline Somalia & 27 \\
\hline Iraq & 15 \\
\hline Vietnam & 8 \\
\hline Sudan & 7 \\
\hline Iran & 7 \\
\hline Afghanistan & 6 \\
\hline Ethiopia & 6 \\
\hline Russia & 4 \\
\hline Other ( 76 countries) & 20 \\
\hline
\end{tabular}

in the U.S. within the past 1-5 years, (2) employees of voluntary resettlement agencies (VOLAGs), (3) personnel of mutual assistance agencies (MAAs), or (4) health care providers (HCPs) with a large refugee clientele base.

\section{Study Definitions and Eligibility}

For the purposes of this study, a 'refugee' was defined as a primary refugee, secondary refugee migrant, asylee, parolee, or victim of human trafficking and the term 'refugee' is used interchangeably with 'resettled refugee' [11]. Study eligibility criteria were: minimum age of 18 years, having knowledge of refugees in San Diego County and their health needs, and willingness and ability to provide voluntary informed consent. In addition, refugee informants were required to have resided in the U.S. for more than one but $<5$ years and to be from one of the top eight countries of origin of recently resettled populations in San Diego. VOLAGs are federally funded to provide resettlement services-e.g., initial contact, housing, food, health care enrollment, employment services, and English as a Second Language (ESL) instruction—during refugees' first 90 days after arrival. VOLAGs, however, generally maintain contact throughout the first year of resettlement and by virtue of their work, remain connected to refugee 
communities. An MAA is a non-profit community-based organization with at least $51 \%$ of their governing board comprised of former or current refugees with the purpose of providing social services for resettlement [12]. Health care providers with a large refugee clientele base were targeted as they are generally especially knowledgeable about refugee health needs and potential barriers involved in treating the refugee population.

\section{Sampling Methods}

In qualitative research, the sampling strategy aims to reflect a diversity of viewpoints, rather than a statistical representation of the population. The minimum number of interviews on a subject needed to identify major themes on an issue is generally agreed to be 15-20 [13]. By conducting 40 interviews, we anticipated identifying most major health care access issues. Firm conclusions regarding differential attitudes/practices across any of the four informant groups or among any of the eight refugee nationality groups, however, were less likely. However, the main aim of this analysis was to identify the perceived health care access barriers affecting this resettled refugee population, regardless of which group identified these barriers.

In order to reflect a diversity of viewpoints targeted sampling methods were used. A list of 111 refugee serving organizations in San Diego County, which included VOLAGs, MAAs, and health care providers, was created by combining information from several local health organizations along with a list of health care providers from the Medi-Cal Provider Directory given to refugees upon resettlement. The list's validity was verified by a brief phone survey.

San Diego has four VOLAGs and each selected one employee who was familiar with refugee health issues to participate. Prior to selection, MAAs were categorized by population served (e.g., Ethiopians) and randomly sampled when more than one organization served a particular group. When contacted, the willing MAAs would suggest the individual to be interviewed based on the assessment objectives. For health care providers $(n=10)$, several large clinics whose catchment area largely consisted of refugees were targeted, with the remaining being health clinics and doctors' offices randomly selected from a health care provider list. Sampling of refugee informants $(n=16)$ was stratified to include one male and one female from each of the eight refugee groups listed above. Recruitment occurred through referrals from MAAs and VOLAGs, and personnel connected with specific refugee communities who were familiar with project aims. Key stakeholders in the community, such as community leaders familiar with concerns within their community, were targeted.

\section{Data Collection}

To optimize rapport, interviewers were matched to respondents based on gender when logistically possible. This was accomplished for all refugee interviews and 63\% of interviews with refugee service and health care providers. Trained interpreters conducted interviews with participants who preferred to express their opinions in their native language. Interviews lasted $\sim 1$-h, were conducted in private locations based on availability and participant preference, were audio taped, translated into English (when necessary), and transcribed. Interviews began with collection of demographic information including age, gender, and educational level. A semi-structured interview guide was then used to elicit information on health conditions of concern to the refugee community, socio-cultural issues surrounding resettlement, health care access, health promotion, and feasibility of conducting future health assessments. Informed consent was received before each interview and study participants were offered \$15 USD as reimbursement for their time. Study methods were reviewed and approved by institutional review boards at the University of California, San Diego and San Diego State University.

Analysis

Transcripts were read and coded by two investigators (MM and SP) to identify themes, trends, and emerging patterns surrounding health care access. In addition to responses to two specific questions regarding health care utilization (What are issues limiting refugees from using health services? Do you know any refugees who needed to see a doctor but did not go? What were the reasons?), health care access issues throughout the interview were coded to fully capture health care utilization. Consistency and agreement on emergent themes were assessed and then clarified for a subsequent round of coding. Atlas.ti software version 5.0 (Berlin, Germany) was used to organize and analyze emergent themes. Descriptive statistics of participants were calculated using the Statistical Package for the Social Sciences for Microsoft Windows version 13.0 (SPSS, Inc., Chicago, 2004).

\section{Results}

Characteristics of Participants

The study population was comprised of a total of 40 individuals from the four target groups. Half of the refugee service providers (VOLAG/MAA) interviewed were college graduates, the majority were male $(57 \%)$, with most 
(12/14) being former refugees themselves (see Table 2). As some non-governmental organizations do not have a long history of working with the refugee community, participants were asked for the number of years their organization had worked with refugees: median 10.5 years, inter-quartile range (IQR) 4.9-22.8.

Due to difficulties in scheduling, only one physician treating refugees on a regular basis could be interviewed. Instead, health care providers included social workers and nurses, with a majority being directors of health promotion programs. Three providers were born outside of the U.S. and one was a former refugee. All but one health care provider had a Masters degree or higher. The median number of years the health care organizations had been serving refugees was 18.5 (IQR 7.5-41).

Refugees were approximately equally likely to have been in the U.S. 1 (25\%), 2 (19\%), 3 (19\%), or 4 years (38\%; Table 3). As outlined in the Methods, refugees were sampled to get an equal representation of both genders from all eight countries of origin. Approximately half of refugee participants were older than 40 years and had no educational experience beyond high school, although $25 \%$ had a college degree or higher.

\section{In-depth Interviews}

\section{Health Conditions Emerging upon Resettlement}

While this analysis is focused on health care utilization, our study revealed a variety of long-term health conditions that provide context for the type of care most needed by the San Diego refugee community. Overall, mental health (informal diagnoses of PTSD, depression, and anxiety) was the most commonly mentioned urgent health concern (Hurst et al., in prep). Hypertension and diabetes were considered emerging problems in the community; however, participants did not discuss in detail the extent to which these conditions are being controlled in those affected. Additionally, dental health is a common refugee health concern upon arrival in the U.S. which persists in the years following resettlement. While few children were considered obese, participants noted that nutritious foods weren't often included in diets and weight gain was more common with years post-resettlement (Rondinelli et al., in prep). Lastly, health concerns specific to women included family planning, under utilization of pre- and postnatal care, and health implications of female genital cutting.

\section{Access to Health Care}

A number of themes emerged in respect to health care access, including transportation and logistical barriers, along with insurance and medical expense. Because these
Table 2 Characteristics of health care provider, mutual assistance agency, and voluntary resettlement agencies participants $(n=24)$

\begin{tabular}{lll}
\hline Category & VOLAG/MAA & $\begin{array}{l}\text { Health care } \\
\text { providers } \\
N=10\end{array}$ \\
\hline $\begin{array}{l}\text { Gender } \\
\quad \text { Male }\end{array}$ & \\
$\begin{array}{l}\text { Age (years) } \\
<39\end{array}$ & 0 \\
$40-54$ & 8 & 3 \\
$\geq 55$ & 5 & 5 \\
Highest education level completed & 2 \\
High school or below & 1 & \\
Some college & 6 & 0 \\
College grad & 5 & 1 \\
Masters & 2 & 0 \\
PhD/MD/JD & 0 & 6
\end{tabular}

No. years organization has served refugees

Median (inter-quartile range) $10.5(4.9,22.8) \quad 18.5(7.5,41.0)$

Populations often served

Non-refugees $\quad 1 \quad 6$

All refugee groups $\quad 3 \quad 1$

Vietnamese $\quad 3 \quad 6$

Sudanese $\quad 7 \quad 4$

Somalis $\quad 9 \quad 5$

Russians $\quad 3 \quad 2$

Iraqis 3

Iranians $\quad 4 \quad 1$

Ethiopians $\quad 6 \quad 3$

$\begin{array}{lll}\text { Afghanis } & 4 & 2\end{array}$

Former refugee

Yes $12 \quad 1$

Country of origin of former refugees $(n=12)$

Albania $\quad 1 \quad 0$

Ethiopia $\quad 2 \quad 0$

Iraq

Poland

Somalia

Sudan

Vietnam

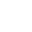

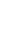

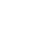

(1)

Services provided

Family medicine $\quad 5$

Internal medicine 3

Dental 4

Gynecological 4

Mental health 5

Geriatrics 4

Pediatrics 4

Resettlement 3

Education 8

Job training 6 
Table 2 continued

\begin{tabular}{lll}
\hline Category & VOLAG/MAA & $\begin{array}{l}\text { Health care } \\
\text { providers } \\
N=10\end{array}$ \\
\hline Legal assistance & 7 & \\
Child care & 4 & \\
Health promotion & 10 & \\
\hline
\end{tabular}

Table 3 Characteristics of refugee participants $(n=16)$

\begin{tabular}{ll}
\hline Category & Number \\
\hline Gender & \\
$\quad$ Male & 8 \\
Age (years) & 2 \\
$\quad<25$ & 5 \\
$25-39$ & 6 \\
$40-54$ & 3 \\
$\geq 55$ & \\
Highest education level completed & 4 \\
Elementary school or below & 2 \\
Middle school & 3 \\
High school & 2 \\
Trade/technical school & 1 \\
Some college & 3 \\
College grad & 1 \\
PhD/MD/JD & \\
Number of years in US & 4 \\
1 Year & 3 \\
2 Years & 3 \\
3 Years & 6 \\
4 to $\leq 5$ years & \\
\hline
\end{tabular}

issues are not specific to refugees and have already received attention in refugee health literature, we only present a brief summary of these themes [14-17]. Instead, we discuss in more depth language and communication along with acculturation barriers and cultural beliefs regarding health care. These were perceived by all groups to be the most immediate and common barriers affecting health care utilization.

Logistical issues, such as transportation, hours of service, wait times, appointment availability, childcare needs, and barriers around scheduling, were a recurrent theme in the interviews. Barriers related to insurance included gaps in coverage due to hurdles with enrollment of public insurance programs or unavailability through one's employment. Financial hardship was also a dominant barrier within this theme-specifically the financial burden of insurance fees, co-payments, and out of coverage prescriptions.

\section{Language and Communication}

Every participant perceived language to be a major, if not the most major barrier to refugee health care access. The extent to which language was as a barrier varied across ethnic groups, with groups more established in the community having less difficulty. For instance, all Vietnamese representatives interviewed noted that language was not a problem because "well, we have a lot of Vietnamese doctors, and we have Vietnamese workers at most of the clinics..." (MAA, former refugee, age 69). However, language was noted as a barrier when Vietnamese patients were referred to a specialist outside the community. For some groups, even when there were health care resources in their native language, the quality of such care was in question. A health care provider described a situation where Russian refugees were killing quality of care to be seen by a provider who spoke the same language. "They actually say 'I know this doctor isn't good, but I'm going to go there because they speak my language'" (HCP, age 31).

Several health care providers mentioned that language barriers do not exist only during interactions between doctors and patients but at every level of the health care system, from making an appointment to filling a prescription. Over half of participants perceived language barriers to lead to refugees either utilizing health care services only when they are very sick, or not at all. Participants agreed that even when translation services were available for the actual doctor's visit "most of the time people [who] answer the phone to schedule an appointment...they don't speak the [same] language" (VOLAG, former refugee, age 31). Refugee and MAA participants also discussed the difficulty refugees have with filling out paperwork and understanding written materials, especially in regards to providing informed consent. For instance, a refugee mentioned that she was unable to write her name when she came to the U.S. and recalls the difficulty she had—and still has—with paperwork. All participant groups shared the view that "A lot of times refugees will get so frustrated that [they don't] bother going to the doctor unless something is really seriously wrong" (VOLAG, former refugee, age 31).

Health care providers spoke more about the effects of low or no English-language proficiency on refugees' understanding of and ability to navigate through the health care system. One provider shared an experience where, due to a lack in proper translation, a Somali refugee delivered her baby on the doorstep of a hospital when there were open after-hour emergency services on the other side of the building. All MAA/VOLAG participants discussed the consequences language barriers have on refugees' ability to properly understand diagnoses and treatment options. Misinterpretation and miscommunications during appointments can go both ways and sometimes have serious 
consequences. For instance, a service provider recalled the case where a father was incorrectly interpreted as having said "Should I throw the baby away?" upon finding out that his baby was diagnosed with hepatitis B. Child protective services were called and an MAA employee had to personally intervene when the father came to them crying that he was going to lose his child. Health care providers also mentioned the impact misinterpretation has on the quality of care they are able to provide, noting how difficult it was to properly diagnose patients when communication was broken and physician time is limited.

Both health care providers and MAA/VOLAGs pointed out that language barriers also affect refugees' ability to fill prescriptions and properly take medicine. One participant shared an experience with a recent refugee taking the wrong medicine all together:

"She told me one day ...she wants to take birth control pills. I said, show me. [She] show me the Tylenol....She said, 'Is this Tylenol or birth control'? ...She doesn't know which one is which..." (VOLAG, age 34).

When discussing interpretation services, almost all refugee participants relied on friends and family to act as interpreters instead of a professional service. A majority of MAA/VOLAG and health care providers perceived problems with relying on family members, including misinterpretation and confidentiality issues.

"I think the language barrier is a big one...making sure someone can translate medically, you know not just translate the words, but make them understand what it is that's needed." (HCP, age 39)

Every participant saw interpretation services as one possible solution to language barriers. However, there was much variation in participants' perceptions of the quality of interpretation services currently available and who is responsible for providing them. Refugee serving organizations (MAA/VOLAG) placed the responsibility on clinics and health care providers. Because they realized it was difficult for providers to provide adequate language services, the majority of MAAs perceived a need for the refugee serving organizations themselves to remedy the language problem - and often expressed the opinion that the refugees would not be able to function as adequately as they do without their help. However, these participants also noted that they are under-staffed, under-funded, and often unable to provide the service due to other demands of their job.

Several health care providers brought up concerns that MAA employees are untrained as interpreters, both in medical terminology and in confidentiality issues, and therefore present the same problems as do family members who act as interpreters. Half of the HCP participants agreed that translation responsibility lies with providers, but noted that many clinics do not follow through on this responsibility. "They just don't want to, it's inconvenient ... and it's a cost to actually have [language services]" (VOLAG, former refugee, age 31 ). The health care providers interviewed are unique in that they work closely with the refugee community on a regular basis and knew about and used interpretation services. However, many mentioned that providers outside the refugee serving community do not always follow this policy. For instance, one provider shared the following conversation of when a hospital worker called him yelling the following:

"Your patients are so incompliant, and we have this patient here now. And she doesn't care that she's going to, you know the kid's going to die ... she doesn't want to sign this letter [consent for putting her child under anesthesia]. ...And we really don't know if we want any more of your patients." (HCP, age 47)

Upon speaking further with the provider the interviewee learned that the hospital was trying to get the patient to consent to a procedure without an interpreter present to explain the situation to her. The patient misunderstood and originally thought the doctor was going to put the child permanently to sleep.

\section{Acculturation Difficulties}

Acculturation as a barrier to utilization of care was brought up both directly and indirectly during all interviews. For the purposes of the current study, acculturation is defined as the merging and adoption of behavior patterns between cultures as a result of prolonged contact. Participants said that cultural and environmental differences make adjustment more difficult for resettled populations. A few MAA participants mentioned that even when refugees are from a country with a similar lifestyle and a similar environment to the host country it is still very "difficult for anyone to jump from one culture right into another" due to the journey they have taken, often involving loss of family and a loss of "identity" within the community, the family, or the workplace. Further, all participants perceived adjustment difficulties to affect the elderly more severely.

"Our elders are really suffering in terms of not knowing where to go [for care]... they do not have relatives you know, ... they stay home, and they're really depressed" (Refugee of Middle Eastern origin, age 37).

Mental health problems in relation to or exacerbated by the acculturation process were perceived to be widespread. 
A few refugee participants discussed stress related depression and "guilt" for leaving their old lives; "everyday you think about back [home]..." (VOLAG, former refugee, age 35). Respondents referred to past traumatic experiences as a source of stress and anxiety, but added on top of this was now the stress of adjusting to the fast pace of life in the U.S. One refugee participant stated, "back home...we don't have... this kind of pressure. The life is so easy back home. You don't have bills, you don't drive..." The elderly, who in many cultures command the most respect, were said to now be isolated due to their lack of family and community ties in the U.S.

Conversely, many perceived that newly resettled refugees work to establish and find a community once in the U.S. MAA, HCP, and refugee participants alike discussed the positive role this 'community' plays in accessing care. Several refugee serving organization and refugee participants expanded by saying that communities support refugees by loaning money to pay for prescriptions and medical costs and by serving as translators, emotional support, and a source of knowledge about how the health care system works. "Meeting people from their country and knowing that there are people like us help us to [acculturate]" (VOLAG, age 34).

\section{Cultural Beliefs Regarding Health Care}

We use here a very broad definition of 'culture', encompassing native belief systems, traditions, upbringing, social norms, and personal values. While maintaining the culture of ones homeland often provides comfort to newly arrived refugees entering a foreign place, it can at times make accessing and navigating their new country's health care system more challenging. We found that culture directly affected refugees' concept of prevention services, independence, expectations of care and stigma around health conditions-all of which influenced their health care choices.

It was a common sub-theme-stated by all refugee serving organization participants and health care providers-that many refugees resettled in San Diego are not fully accustomed to the idea of preventive care, such as annual medical, vision, and dental exams; they elaborated that refugees often wait to seek care until conditions are severe. Many HCPs cited the difference between economic and health systems in the host country and a refugee's native country as a reason for under-utilization. For instance, one HCP stated "they had a whole different way of taking care of issues back home...they went to the hospital only when they were sick", accessing health services for prevention was not commonly available to the majority of refugee groups in their home country.
Cultural beliefs and previous health care experiences contributed to refugees' expectations of western medical care. Expectations included: specific behaviors of doctors, curing chronic diseases instead of managing them, and fast care by clinics. A refugee participant shared, "when I go to the doctor I want him to touch me, to look in my eye, hit me with the little hammer..." A few former refugee MAA participants and one refugee participant explained how frustrated and disappointed they were when their health did not immediately improve upon arrival in the U.S. This disappointment made many reluctant to seek care because their expectations were not met:

"We came here with hope to get better right away, so when we seek treatment and we don't get [better], we feel frustrated...we came here with [the] hope that all these problems we have [will] go away. So we...[become] disappointed." (Refugee of African origin, age 35 )

Health care providers in-turn perceived that refugees' culture contributes to a negative perspective of western medicine. Four of the ten health care providers interviewed shared views on how this theme created a barrier to providing care, stating that "the cultural concept of medicine" was different for refugee groups. These providers made references to refugees relying on homeopathic treatments and spiritual healers. However, refugee participants did not view use of homeopathic remedies or use of spiritual healers as a barrier to accessing medical services here in the U.S. Instead, refugee participants noted that they use some cultural remedies but do not view it as a replacement to western care.

Expectations about health care extended to the gender of health care providers. Participants felt a strong yet easily resolvable barrier related to culture was gender matchingespecially for female patients preferring to be seen by female health care providers. Six of the eight female refugee participants and one male noted the importance of same sex care due to cultural and religious beliefs of some refugee populations. Health care providers were also aware of the need for gender matching, especially for reproductive health concerns.

All groups noted how cultural barriers affected utilization of mental health services. Most perceived mental health services to be extremely under-utilized by refugees-yet of great need. Stigma and lack of understanding of what mental health conditions are has made it difficulty to serve the refugee population.

"They think 'mental problems' that...those words should be reserved for... somebody with severe Down's syndrome or something. They don't think mental health can be things like depression or 
posttraumatic stress disorder." (MAA, former refugee, age 24)

Stigma surrounding mental health conditions was brought up by a quarter of refugee participants, and the majority of MAA and VOLAG respondents when discussing mental health care.

"They can even just go ahead and die before they tell somebody that they have a problem that will affect their life for a long time." (MAA, former refugee, age 42)

Even if a person sought mental health care, health care providers who worked in the mental health field acknowledged a great need for more "culturally and linguistically appropriate mental health [services]".

\section{Discussion}

While previous studies of resettled refugee health issues have largely focused on health upon arrival in a host country, we investigated access to care issues in the first years post-resettlement. Despite high expectations by refugees for improved health and health care, our assessment in San Diego County found that many resettled refugees are still not receiving adequate care. Barriers relating to language, the acculturation process, and cultural beliefs regarding health care all contributed to a decreased ability or willingness to obtain care. Many of these barriers are inter-related and jointly contribute to a larger access to care problem among the refugee population. This assessment highlighted both individual and structural-level areas that can be targeted for future studies and intervention.

Consistent with existing literature, language and miscommunication between refugees and their health care providers were perceived to be the most limiting health care access barriers [18-21]. The current study found that language issues do not only affect access to care and basic doctor/patient interactions, but also limit a patient's ability to read and understand medical instructions and prescriptions. This can translate into misuse of medicine and lead to additional health problems. Language barriers have also been found to decrease patients' access to preventive services, such as cancer screening tests [22].

Over the course of resettlement a predominance of acute to more chronic diseases has been documented [3, 23, 24]. Although the factors behind this shift are not fully understood, it is thought that stress from forced migration increases susceptibility to chronic diseases, ranging from cancer, diabetes, hypertension, to mental illness [23, 25]. Additionally, within the resettlement environment, changes in diet or activity level, stress associated with acculturation, and lack of access to or utilization of health services are hypothesized to contribute to chronic disease [20, 23, 26, 27].

Suggestions for alleviating language barriers included increasing the quality of interpretation services through training, improved utilization of AT\&T language line services (a service which provides over the phone interpretation services, without an appointment) and language banks (trained face-to-face interpreters hired through a professional service). Additionally, video interpretation services is an emerging service using video conference call technology to allow communication to flow more freely between patient, provider, and skilled interpreter [28]. Study participants also suggested increasing the availability of English language classes for refugees to decrease the communication health access barrier as well as increase the adaptive advantage of employment and acculturation.

Consistent with recent health literature, refugees' history of migration and changes in social identity and their role within the community post-resettlement were perceived to indirectly affect health access utilization [21]. Even though federal refugee resettlement programs provide aid for refugees during the initial months post-resettlement, more support for social and economic assistance programs would likely be necessary to lessen acculturation barriers. As Palinkas et al. demonstrated, acculturation is a two-way process affecting both the migrant and the organizations dedicated to helping the migrant [29]. Therefore, programs and organizations need to readjust their promotion aims and take into account the cultural and linguistic needs of refugee groups to provide better care.

Participants perceived that preventive care was underutilized by refugees post-resettlement. Similar findings were shown in a qualitative study examining barriers to accessing care of Southeast Asian immigrants. A qualitative study examining barriers to care of Southeast Asian immigrants found cultural attitudes toward suffering, sources of illness were barriers to Western health care service utilization [20]. Cultural beliefs surrounding gender affected the comfort-level of women from many refugee groups in seeking care from health care providers of the opposite sex [30]. Studies have indicated that culturally sensitive prenatal care improves access to and utilization of that care $[30,31]$. Therefore, it is imperative that health care providers working with the refugee population are culturally sensitive and also inform their patients of treatment and care options, when available — which should help empower refugees in their interactions with the health care system. Cultural barriers are not generalizable to the greater immigrant population, nor across refugee groups. Instead they encompass cultural and religious beliefs as well as life experiences that shape their perception of the world. Education programs that train health care providers 
in cultural competency have been shown to improve patient-provider interaction [32].

This assessment was limited in size, in part due to its qualitative design, but also because of resource and time restrictions. Qualitative studies, however, provide valuable data upon which future assessments and health promotion activities can be built. The design of the study, which included several key representatives from each of the 8 largest recently resettled refugee groups in San Diego, precluded us from determining health issues specific to any particular ethnic group. Further, our findings cannot be generalized to resettled refugees living in other parts of the world. However, many of the health access themes identified appeared to be common concerns of resettled groups [19, 33-35]. Since all health care providers interviewed were women there is a possibility that certain perspectives were not included. Furthermore, because all health care providers regularly treated refugees, they provided a different view of refugees' health care access needs than one might find from a HCP who is less familiar with refugees. This is most likely why there were few differences between perceived health care access barriers by HCP and VOLAG/ MAA participants.

There is limited literature available on the relationship between health access and refugee long-term health outcomes within resettled countries [33]. This is a population which has been forced from their homes, and undergone tremendous hardship prior to resettlement within a host country-a background which creates specific health needs. In order to better allocate resources and serve this population more effectively, population-specific research and evaluation of existing programs would likely improve care.

Acknowledgments We sincerely thank the study participants who gave their time and shared their opinions, beliefs, and experiences. We gratefully acknowledge the help of the San Diego Refugee Forum, and refugee community members. Many thanks to the interpreters and interviewers who made this assessment possible. Special thanks to Kathleen Moser, M.D., M.P.H., and Paulino S. Paida from the County of San Diego Health and Human Services Agency. The work was funded by the County of San Diego Health and Human Services Agency grant \#06-37-9361-01.

Open Access This article is distributed under the terms of the Creative Commons Attribution Noncommercial License which permits any noncommercial use, distribution, and reproduction in any medium, provided the original author(s) and source are credited.

\section{References}

1. Division of Operational Services Field Information and Coordination Support Section UNHCofR. (2007). 2006 Global trends; refugees, asylum-seekers, returnees, internally displaced and stateless persons, Geneva.
2. Stauffer, W. M., Kamat, D., \& Walker, P. F. (2002). Screening of international immigrants, refugees, and adoptees. Primary Care, 29(4), 879-905.

3. Ackerman, L. K. (1997). Health problems of refugees. Journal of the American Board of Family Practice, 10(5), 337-348.

4. Lifson, A. R., Thai, D., O'Fallon, A., Mills, W. A., \& Hang, K. (2002). Prevalence of tuberculosis, hepatitis B virus, and intestinal parasitic infections among refugees to Minnesota. Public Health Report, 117(1), 69-77.

5. Miller, J. M., Boyd, H. A., Ostrowski, S. R., Cookson, S. T., Parise, M. E., Gonzaga, P. S., et al. (2000). Malaria, intestinal parasites, and schistosomiasis among Barawan Somali refugees resettling to the United States: A strategy to reduce morbidity and decrease the risk of imported infections. American Journal of Tropical Medicine and Hygiene, 62(1), 115-121.

6. Varkey, P., Jerath, A. U., Bagniewski, S. M., \& Lesnick, T. G. (2007). The epidemiology of tuberculosis among primary refugee arrivals in Minnesota between 1997 and 2001. Journal of Travel Medicine, 14(1), 1-8.

7. Toole, M. J., \& Waldman, R. J. (1997). The public health aspects of complex emergencies and refugee situations. Annual Review of Public Health, 18, 283-312.

8. Tiong, A. C., Patel, M. S., Gardiner, J., Ryan, R., Linton, K. S., Walker, K. A., et al. (2006). Health issues in newly arrived African refugees attending general practice clinics in Melbourne. Medical Journal of Australia, 85(11-12), 602-606.

9. San Diego County Health and Human Services Agency. (2006). San Diego arrivals by groups and nationalities, 2001-2005. San Diego: California Department of Health Services Refugee Health Section.

10. San Diego County Health and Human Services Agency. (20002005). RHEIS data.

11. San Diego Refugee Forum. (2006). 2005-2006 San Diego refugee forum interest survey.

12. California Department of Social Services: Refugee Programs Bureau. (2006). MAAs fact sheet.

13. Strauss, A., \& Corbin, J. (1998). Basics of qualitative research: Techniques and procedures for developing grounded theory (2nd ed.). Thousand Oaks: Sage.

14. Silka, L., Toof, R., Liang, S., et al. (2008). Building on the strengths of a Cambodian refugee community through community-based outreach. Health Promotion Practice, 9(4), 415-425.

15. Herrel, N., Olevitch, L., DuBois, D. K., Terry, P., Thor, D., Kind, E., et al. (2004). Somali refugee women speak out about their needs for care during pregnancy and delivery. Journal of Midwifery Womens Health, 49(4), 345-349.

16. Pesata, V., Pallija, G., \& Webb, A. A. (1999). A descriptive study of missed appointments: Families' perceptions of barriers to care. Journal of Pediatric Health Care, 13(4), 178-182.

17. Yang, S., Zarr, R. L., Kass-Hout, T. A., Kourosh, A., \& Kelly, N. R. (2006). Transportation barriers to accessing health care for urban children. Journal of Health Care for Poor and Underserved, 17(4), 928-943.

18. Sheikh-Mohammed, M., Macintyre, C. R., Wood, N. J., Leask, J., \& Isaacs, D. (2006). Barriers to access to health care for newly resettled sub-Saharan refugees in Australia. Medical Journal of Australia, 185(11-12), 594-597.

19. Lipson, J. G., \& Omidian, P. A. (1992). Health issues of Afghan refugees in California. Western Journal of Medicine, 157(3), 271-275.

20. Uba, L. (1992). Cultural barriers to health care for Southeast Asian refugees. Public Health Reports, 107(5), 544-548.

21. Segal, U. A., \& Mayadas, N. S. (2005). Assessment of issues facing immigrant and refugee families. Child Welfare, 84(5), 563-583. 
22. Woloshin, S., Schwartz, L. M., Katz, S. J., \& Welch, H. G. (1997). Is language a barrier to the use of preventive services? Journal of General Internal Medicine, 12(8), 472-477.

23. Palinkas, L. A., \& Pickwell, S. M. (1995). Acculturation as a risk factor for chronic disease among Cambodian refugees in the United States. Social Science \& Medicine, 40(12), 1643-1653.

24. Steel, Z., Silove, D., Phan, T., \& Bauman, A. (2002). Long-term effect of psychological trauma on the mental health of Vietnamese refugees resettled in Australia: A population-based study. Lancet, 360(9339), 1056-1062.

25. Carlsson, J. M., Olsen, D. R., Mortensen, E. L., \& Kastrup, M. (2006). Mental health and health-related quality of life: A 10-year follow-up of tortured refugees. The Journal of Nervous and Mental Disorders, 194(10), 725-731.

26. Lears, L. O., \& Abbott, J. S. (2005). The most vulnerable among us. Health Progress, 86(1):22-25, 60.

27. Popkin, B. M., \& Udry, J. R. (1998). Adolescent obesity increases significantly in second and third generation U.S. immigrants: The National Longitudinal Study of Adolescent Health. Journal of Nutrition, 128(4), 701-706.

28. Hirsch, J., \& Marano, F. (2007). Better patient care through video interpretation: A New Jersey hospital uses teleconferencing tools and interpreters to break down patient language and hearing barriers. Health Management Technology, 28(3), 30-31, 37.
29. Palinkas, L. A., Pickwell, S. M., Brandstein, K., Clark, T. J., Hill, L. L., Moser, R. J., et al. (2003). The journey to wellness: Stages of refugee health promotion and disease prevention. Journal of Immigrant Health, 5(1), 19-28.

30. Beine, K., Fullerton, J., Palinkas, L., \& Anders, B. (1995). Conceptions of prenatal care among Somali women in San Diego. Journal of Nurse-Midwifery, 40(4), 376-381.

31. Bell, S. E., \& Whiteford, M. B. (1987). Tai Dam health care practices: Asian refugee women in Iowa. Social Science and Medicine, 24(4), 317-325.

32. Griswold, K., Zayas, L. E., Kernan, J. B., \& Wagner, C. M. (2007). Cultural awareness through medical student and refugee patient encounters. Journal of Immigrant and Minority Health, $9(1), 55-60$.

33. Beiser, M. (2006). Longitudinal research to promote effective refugee resettlement. Transcultural Psychiatry, 43(1), 56-71.

34. Lie, B. (2002). A 3-year follow-up study of psychosocial functioning and general symptoms in settled refugees. Acta Psychiatrica Scandinavica, 106(6), 415-425.

35. Erwin, P., Leung, Y. Y., \& Boban, D. I. (2001). Bosnian refugees in San Francisco: A community assessment. International Institute of San Francisco. 